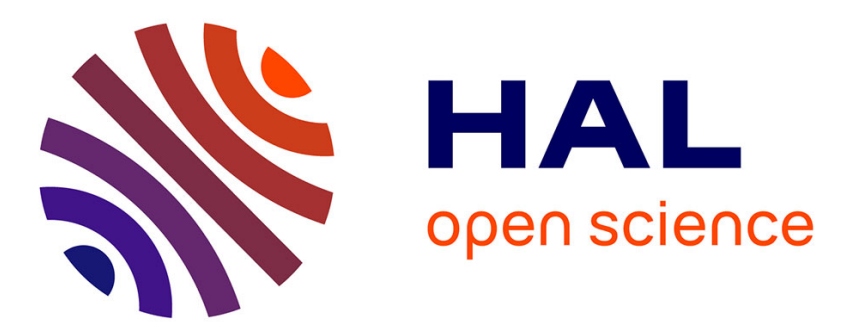

\title{
Carrière des vaches laitières: Caractérisation de la phase d'élevage et relation avec les performances en première lactation
}

\author{
J.L. Troccon, J.B. Coulon, F. Lescourret
}

\section{- To cite this version:}

J.L. Troccon, J.B. Coulon, F. Lescourret. Carrière des vaches laitières: Caractérisation de la phase d'élevage et relation avec les performances en première lactation. Productions Animales, 1994, 7 (5), pp.359-368. hal-00896097

\section{HAL Id: hal-00896097 https://hal.science/hal-00896097}

Submitted on 1 Jan 1994

HAL is a multi-disciplinary open access archive for the deposit and dissemination of scientific research documents, whether they are published or not. The documents may come from teaching and research institutions in France or abroad, or from public or private research centers.
L'archive ouverte pluridisciplinaire HAL, est destinée au dépôt et à la diffusion de documents scientifiques de niveau recherche, publiés ou non, émanant des établissements d'enseignement et de recherche français ou étrangers, des laboratoires publics ou privés. 
INRA Prod. Anim., 1994,7 (5), $359-368$

\section{J.L. TROCCON, J.B. COULON*, F. LESCOURRET $T^{* * \%}$}

INRA Station de Recherches sur la Vache Laitière 35590 Saint-Gilles

* INRA Laboratoire Adaptation des Herbivores aux Milieux

Theix 63122 Saint-Genès Champanelle

** INRA Laboratoire d'Ecopathologie. Theix 63122 Saint-Genès Champanelle.

\section{Carrière des vaches laitières : \\ Caractérisation de la phase d'élevage et relation avec les performances en première lactation.}

La carrière d'une vache laitière débute par la phase d'élevage de la génisse qui dure généralement entre 2 et 3 ans pour une durée de vie productive d'environ 4 ans. Au cours de cette phase, les génisses acquièrent leur maturité sexuelle et une grande part de leur développement corporel. Leur alimentation pendant cette période (avant et après la puberté) a des conséquences sensibles sur la production laitière des adultes (Troccon et Petit 1989). La phase d'élevage des génisses est donc de première importance. L'étude des conséquences de conduites différentes sur les performances des vaches nécessite de disposer d'effectifs importants sur une longue période.

\section{Résumé}

Les courbes de croissance de 971 génisses qui ont vêlé pour la première fois entre 1967 et 1991 dans les domaines expérimentaux INRA de Marcenat (Cantal), Theix et Orcival (Puy de Dôme) sont analysées ainsi que leurs conséquences sur les performances au début de la lère lactation. Ces génisses des races Montbéliarde, Frisonne, Holstein et les 2 croisements Holstein ont vêlé pour la première fois vers l' âge de 2 ans ( 274 vêlages précoces) ou de 3 ans (697 vêlages tardifs). Le poids vif après le premier vêlage des primipares précoces est plus faible de $83 \mathrm{~kg}$ et leur production laitière ajustée est réduite de $2 \mathrm{~kg} / \mathrm{j}$. Les cas de mortalité des veaux produits, de soins génitaux et de mammites sont accrus chez les vaches primipares précoces. Ces 2 populations ont été analysées séparément.

Les profils de croissance issus de la classification des génisses selon leurs gains de poids vifs (4 pour les primipares précoces et 7 pour les primipares tardives) conduisent à des poids vifs après le premier vêlage de 470 à $490 \mathrm{~kg}$ pour les vaches primipares précoces et de 510 à $610 \mathrm{~kg}$ pour les vaches primipares tardives. Les différences maximales de poids vif étaient de $45 \mathrm{~kg}$ à l'âge de 6 mois et de $81 \mathrm{~kg}$ à l'âge de 12 mois. La différence de profil de croissance des vaches primipares précoces (4 profils identifiés) n'a pas d'incidence sur la production laitière au début de la première lactation. La production laitière des vaches primipares tardives augmente de $1,8 \mathrm{~kg} / \mathrm{j}$ lorsque leur poids vif après le premier vêlage augmente de 530 à $590 \mathrm{~kg}$. Le profil de croissance recommandé pour les génisses primipares tardives pourrait présenter des croîts modérés de la naissance aux âges de 6 ou 12 mois (700 $\mathrm{g} / \mathrm{j})$ puis jusqu'après le premier vêlage $(400 \mathrm{~g} / \mathrm{j})$ sans pénaliser l'expression $\mathrm{du}$ potentiel laitier. Ces valeurs sont dans la moyenne des gains de poids vif de notre population de génisses primipares tardives.
Un haut niveau d'alimentation avant la puberté conduit généralement à une réduction de la production laitière (Hansson et al 1967, Amir et al 1968, Little et Kay 1979, Waldo et al 1988, Foldager et Sejrsen 1991). A partir du $3^{\text {eme }}$ mois d'âge et au cours de la période prépubère (Sinha et Tucker 1969), les canaux mammaires s'allongent et se ramifient dans le tissu adipeux de la mamelle (Jammes et Djiane 1988). A ce stade, accroître les apports énergétiques réduit ce développement par suite d'une moindre stimulation hormonale (Sejrsen et al 1983, Capuco et al 1988) et d'une maturité sexuelle plus précoce (Troccon et Petit 1989). La mamelle contient alors moins de tissu sécréteur même après plusieurs lactations (Harrisson et al 1983). Le gain de poids vif prépubère des génisses de race de grand format doit être inférieur à $800 \mathrm{~g} / \mathrm{j}$ (Amir et Kali 1975) voire proche de $600 \mathrm{~g} / \mathrm{j}$ (Foldager et Sejrsen 1991). Au contraire, la production laitière des vaches primipares précoces augmente avec le niveau d'alimentation après la puberté (Foldager et Sejrsen 1991). En effet, les développements du tissu sécréteur mammaire, du format et des réserves corporelles des génisses sont accrus par un haut niveau d'alimentation. Cependant, la production laitière n'augmente plus lorsque le gain de poids vif en fin de gestation augmente de 800 à $1000 \mathrm{~g} / \mathrm{j}$ (Ducker et al 1985, Lacasse et al 1993).

Cet article présente une étude réalisée à partir de la base de données LASCAR (Coulon et al 1993) qui regroupe les caractéristiques et les performances (production, poids vif, reproduction, santé) à l'échelle de la vie de 1179 vaches conduites dans les domaines expérimentaux INRA de Marcenat (Cantal), Theix et Orcival (Puy de Dôme), qui ont vêlé pour la première fois entre 1967 et 1991. L'objectif est de décrire la croissance des génisses entre leur naissance et leur premier vêlage et d'en analyser les conséquences sur les performances au début de la première lactation. 
Figure 1. Répartition des génisses selon leurs caractéristiques.
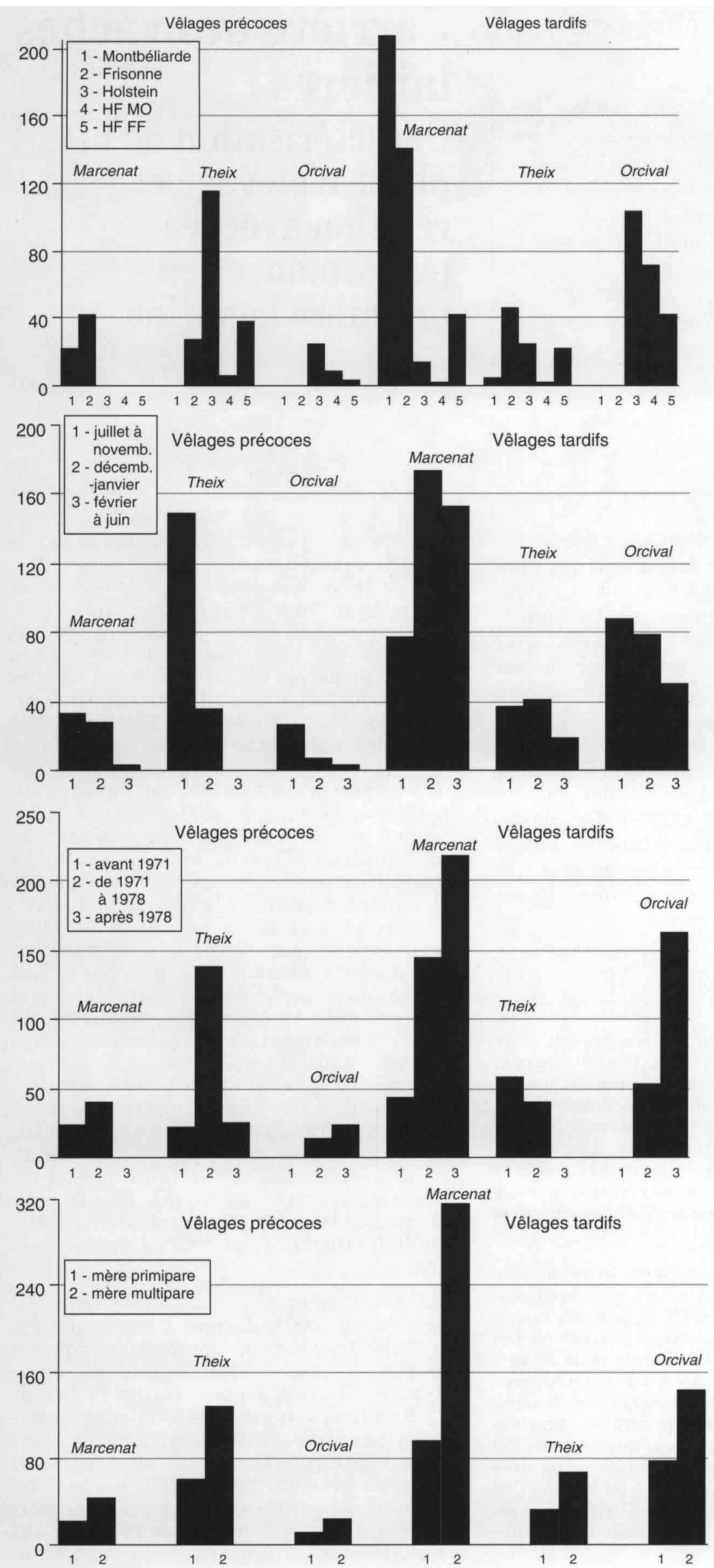

\section{1 / Conduite de l'étude}

\section{1 / Animaux}

Parmi les 1179 vaches, 108 ont été écartées en raison de données incomplètes concernant le poids vif et 5 pour un premier vêlage très tardif ( 4 ans). Les 56 génisses nées jumelles et les 39 génisses à première gestation gémellaire ont fait l'objet d'une analyse séparée succincte (annexe 1). La population restante étudiée (971 génisses) présente une répartition bimodale très nette de l'âge au premier vêlage, centrée sur 24,5 mois (vêlages précoces, à 2 ans, $\mathrm{n}=274$ ) et 35,2 mois (vêlages tardifs, à 3 ans, $n=697$ ), qui reflète les 2 conduites d'élevage pratiquées selon les domaines et les périodes. En conséquence, l'analyse des données a été réalisée séparément pour les 2 populations.

Dans les 3 domaines, les vêlages des génisses ont eu lieu en automne. Le sevrage, la première mise à l'herbe et les traitements sanitaires ont été réalisés dans des conditions similaires. Les génisses ont été sevrées au cours de la 9ème semaine en moyenne et avant l'âge de 3 mois pour $90 \%$ de l'effectif. Après la suppression du lait de remplacement, l'apport journalier d'aliment concentré a été limité à 2-2,5 kg bruts par génisse ; les fourrages ont été offerts à volonté. Dans la majorité des cas, les génisses ont pâturé l'été suivant leur naissance et ont fait l'objet de prophylaxies sanitaires (vaccination contre le charbon, traitements anti-parasitaires, ...). Les génisses ont toujours été logées sur paille en étable entravée ou en stabulation libre. Dès la fin des années 60 , la mise à la reproduction est le plus souvent intervenue après synchronisation des cycles sexuels (traitement Synchro-Mate B).

En hiver, le fourrage distribué aux génisses sevrées a varié selon les domaines. A Marcenat (1100 m d'altitude), les fourrages ont été des foins, des regains et des ensilages d'herbe de prairies permanentes, parfois des pailles de céréale. A Theix $(850 \mathrm{~m})$, la proximité de la Limagne a permis, dès la fin des années 60 , de proposer d'excellents ensilages de maïs à l'ensemble des génisses dès le premier hiver, et en particulier à celles destinées à un vêlage précoce. Les génisses destinées à un vêlage tardif ont reçu essentiellement de la paille de céréale à partir du deuxième hiver. A Orcival $(1000 \mathrm{~m})$, les génisses ont souvent reçu de bons ensilages d'herbe mais aussi des fourrages pauvres ayant subi ou non différents traitements chimiques (soude, ammoniaque). Au début de la lactation, les vaches primipares ont progressivement reçu de l'aliment concentré en fonction de leur production laitière d'abord attendue puis observée et d'un éventuel besoin de croissance (vêlages précoces).

\section{2 / Données et analyses}

Les variables retenues dans cette étude concernent les conditions de naissance des génisses qui les caractérisent intrinsèquement, les gains de poids vif et les poids vifs entre la naissance et le premier vêlage et les performances au début de la première lactation 
(16 semaines). Les conditions de naissance englobent le domaine d'origine (Marcenat, Theix, Orcival), la race (Montbéliarde : MO, Française Frisonne Pie Noir : FF, Holstein : HF, Holstein $x$ Montbéliarde : HFMO, Holstein x Frisonne Pie Noir : HFFF), le rang de lactation de la mère ( 1 ou 2 et plus), la période de naissance (avant 1971, de 1971 à 1978, après 1978) qui repose sur l'introduction du sang Holstein à Theix au début des années 70 puis à Marcenat à la fin des années 70 , et la saison de naissance (juillet à novembre, décembre et janvier, février à juin).

Les poids vifs à 4 âges définissent la courbe de croissance des génisses : à la naissance, aux âges de 6 mois et 12 mois, dans la semaine après le premier vêlage. De la naissance à l'âge de 6 mois, la croissance squelettique est élevée et le développement musculaire important. Le poids à l'âge de 12 mois est proche de celui de la puberté, au moins pour les génisses pie noir. Les poids vifs aux âges de 6 et 12 mois ont été calculés par interpolation des poids mesurés régulièrement au cours de la phase d'élevage. Le poids après le premier vêlage correspond aux pesées réalisées au cours de la première semaine de la $1^{\text {ère }}$ lactation (jours 1 à 7 ) et situe le niveau de développement atteint par la génisse à la fin de la phase d'élevage.

Les profils de croissance des génisses ont été élaborés, pour chacune des 2 populations identifiées (correspondant respectivement aux vêlages précoces et tardifs) à l'aide d'une analyse en composantes principales suivie d'une classification ascendante hiérarchique. Les variables introduites dans ces analyses ont été les gains de poids vif de la naissance à 6 mois, de 6 à 12 mois et de 12 mois au premier vêlage qui sont peu corrélées entre eux $(0,03<\mathrm{r}<0,44)$ contrairement aux poids vifs $(0,49<\mathrm{r}<0,75)$.

L'analyse des relations entre ces profils de croissance et les caractéristiques des animaux a été réalisée de manière à tenir compte des fortes liaisons entre ces caractéristiques. Les liaisons sont particulièrement marquées entre le domaine d'origine et la race ou le mois de naissance (figure 1). Pour cela, un tableau de contingence croisant en ligne les combinaisons de ces caractéristiques présentant un effectif supérieur ou égal à 10 , et en colonne les profils de croissance a été construit. Ce tableau a été soumis à une classification hiérarchique sur distance du Khi2 afin de regrouper les différentes combinaisons en un petit nombre de groupes homogènes à l'égard du profil de croissance.

Les performances au début de la première lactation sont le poids à la naissance des veaux produits, la production laitière journalière (initiale des jours 4,5 et 6 de la lactation, maximale hebdomadaire, au cours des 16 premières semaines de la lactation) et les problèmes sanitaires (mortalité des veaux, rétentions placentaires, soins génitaux après le vêlage, mammites au cours de la première semaine de la lactation). Les relations entre les profils de croissance des génisses et les performances en début de première lactation ont été étudiées par analyse de variance pour les variables quantitatives (lait, poids des veaux, etc ; procédure GLM de SAS, 1987) et par le test du Khi2 pour les variables qualitatives (troubles sanitaires, etc ; procédure FREQ de SAS, 1987).

\section{2 / Résultats}

Les génisses primipares à 2 ans sont nées 7 semaines plus tôt dans l'année que celles vêlant à 3 ans, mais leur premier vêlage est intervenu 9 jours plus tôt (tableau 1). Les poids à la naissance et aux âges de 6 et 12 mois des 2 populations ont été peu différents ainsi que les gains de poids vif entre la naissance et 1 an $(740 \mathrm{~g} / \mathrm{j})$. De l'âge de 1 an jusqu'après le premier vêlage, les génisses

Tableau 1. Performances des génisses et des vaches selon l'âge au ter $^{\text {vêlage. }}$

\begin{tabular}{|c|c|c|c|c|}
\hline Premier vêlage & Précoce & & tardif & \\
\hline Effectif & 274 & & 697 & \\
\hline Date de naissance & 18 Nov. & & 06 Jan. & \\
\hline Date du 1er vêlage & $30 \mathrm{Nov}$ & & 09 Déc. & \\
\hline Age au 1er vêlage (mois) & $24,5 \pm 1,5$ & & $35,2 \pm 2,0$ & \\
\hline Gain de poids vif $(\mathrm{g} / \mathrm{j})$ & & & & \\
\hline naissance - 6 mois & $751 \pm 103$ & a & $730 \pm 104$ & a \\
\hline 6 mois - 12 mois & $679 \pm 104$ & $\mathrm{a}$ & $669 \pm 124$ & $\mathrm{~b}$ \\
\hline 12 mois - après ler vêlage & $487 \pm 96$ & a & $386 \pm 60$ & $\mathrm{~b}$ \\
\hline \multicolumn{5}{|l|}{ Poids vif (kg) } \\
\hline naissance & $37,6 \pm 6,1$ & a & $39,2 \pm 6,1$ & a \\
\hline 6 mois & $172 \pm 20$ & a & $170 \pm 20$ & $\mathrm{a}$ \\
\hline 12 mois & $294 \pm 28$ & a & $290 \pm 30$ & $\mathrm{~b}$ \\
\hline après 1er vêlage & $479 \pm 36$ & a & $562 \pm 50$ & $\mathrm{~b}$ \\
\hline \multicolumn{5}{|l|}{ Première gestation } \\
\hline Durée (jours) & $280 \pm 6$ & a & $283 \pm 7$ & $\mathrm{~b}$ \\
\hline \multicolumn{5}{|l|}{ Poids des veaux simples (kg) } \\
\hline - Mâles & $37,4 \pm 6,5$ & a & $41,1 \pm 5,7$ & $\mathrm{~b}$ \\
\hline - Génisses & $34,8 \pm 5,4$ & a & $37,5 \pm 5,6$ & $\mathrm{~b}$ \\
\hline \multicolumn{5}{|l|}{ Veau/mère après le vêlage $(\%)$} \\
\hline - Mâles & $7,9 \pm 1,3$ & a & $7,4 \pm 1,1$ & $\mathrm{~b}$ \\
\hline - Génisses & $7,2 \pm 1,1$ & a & $6,7 \pm 1,0$ & $\mathrm{~b}$ \\
\hline \multicolumn{5}{|l|}{ Santé au 1er vêlage } \\
\hline Mortalité des veaux $(\%)$ & 13,5 & a & 8,9 & $\mathrm{~b}$ \\
\hline Rétentions placentaires $(\%)$ & 10,2 & a & 8,3 & a \\
\hline Soins après le ler vêlage $(\%)$ & 23,4 & $\mathrm{a}$ & 6,7 & $\mathrm{~b}$ \\
\hline Mammites (\%) & 12,8 & a & 8,0 & $\mathrm{~b}$ \\
\hline \multicolumn{5}{|l|}{ Production en première lactation $(\mathrm{kg} / \mathrm{j})$} \\
\hline Ajustée (2) & 13,7 & $\mathrm{a}$ & 15,5 & $\mathrm{~b}$ \\
\hline maximale (observée) & $20,8 \pm 5,3$ & & $21,5 \pm 5,4$ & \\
\hline Ajustée (2) & 19,8 & a & 21,9 & $b$ \\
\hline en 16 semaines (observée) & $18,4 \pm 4,3$ & & $18,9 \pm 4,5$ & \\
\hline Ajustée (2) & 17,4 & a & 19,5 & $\mathrm{~b}$ \\
\hline Poids adulte $(\mathrm{kg})(3)$ & $622 \pm 71$ & $\mathrm{a}$ & $630 \pm 60$ & $\mathrm{~b}$ \\
\hline nombre de vaches & 170 & & 336 & \\
\hline
\end{tabular}

(1) Les valeurs indexées d'une lettre différente sont statistiquement différentes $(\mathrm{P}<0,05)$.

(2) Productions laitières ajustées par analyse de variance : domaine, race, période de naissance, saison de naissance, âge au premier vêlage.

(3) Poids vif le plus élevé mesuré dans la semaine suivant le vêlage des vaches réalisant au moins 3 lactations. 
Tableau 2. Performances des génisses et vaches primipares précoces selon les profils (1).

\begin{tabular}{|c|c|c|c|c|c|c|c|c|}
\hline Profil & A2 & & B2 & & $\mathrm{C} 2$ & & D2 & \\
\hline $\begin{array}{l}\text { Effectif } \\
\text { Date de naissance } \\
\text { Date du 1er vêlage } \\
\text { Age au 1er vêlage (jours) }\end{array}$ & $\begin{array}{r}52 \\
15 \mathrm{Nov} \\
08 \text { Déc } \\
753,4\end{array}$ & & $\begin{array}{r}117 \\
15 \text { Nov } \\
02 \text { Déc } \\
748,2\end{array}$ & & $\begin{array}{r}57 \\
23 \text { Nov } \\
03 \text { Déc } \\
739,9\end{array}$ & & $\begin{array}{r}48 \\
25 \mathrm{Nov} \\
17 \mathrm{Nov} \\
721,8\end{array}$ & \\
\hline $\begin{array}{l}\text { Gain de poids vif }(\mathrm{g} / \mathrm{j}) \\
\text { Naissance - } 6 \text { mois } \\
\text { De } 6 \text { mois à } 12 \text { mois } \\
\text { De } 12 \text { mois au ler vêlage }\end{array}$ & $\begin{array}{l}776 \\
810 \\
401\end{array}$ & $\begin{array}{l}\mathrm{a} \\
\mathrm{a} \\
\mathrm{a}\end{array}$ & $\begin{array}{l}806 \\
670 \\
462\end{array}$ & $\begin{array}{l}b \\
b \\
b\end{array}$ & $\begin{array}{l}627 \\
680 \\
520\end{array}$ & $\begin{array}{l}c \\
b \\
c\end{array}$ & $\begin{array}{l}734 \\
560 \\
601\end{array}$ & $\begin{array}{l}\mathrm{d} \\
\mathrm{c} \\
\mathrm{d}\end{array}$ \\
\hline $\begin{array}{l}\text { Poids vif (kg) } \\
\text { A la naissance } \\
\text { A l'âge de } 6 \text { mois } \\
\text { A l'âge de } 12 \text { mois } \\
\text { Après le ler vêlage }\end{array}$ & $\begin{array}{r}36,6 \\
175,5 \\
321,3 \\
477,3\end{array}$ & $\begin{array}{l}\mathrm{a} \\
\mathrm{a} \\
\mathrm{abc}\end{array}$ & $\begin{array}{r}37,1 \\
181,4 \\
301,9 \\
480,2\end{array}$ & $\begin{array}{l}\mathrm{b} \\
\mathrm{b} \\
\mathrm{ab}\end{array}$ & $\begin{array}{r}38,2 \\
150,5 \\
272,8 \\
469,8\end{array}$ & $\begin{array}{l}c \\
c \\
b\end{array}$ & $\begin{array}{r}39,3 \\
170,7 \\
271,6 \\
488,8\end{array}$ & $\begin{array}{l}\mathrm{d} \\
\mathrm{c} \\
\mathrm{c}\end{array}$ \\
\hline $\begin{array}{l}\text { Première gestation } \\
\text { Durée (jours) } \\
\text { Poids des veaux simples (kg) } \\
\text { - Mâles } \\
\text { - Génisses } \\
\text { Veau/mère après le vêlage (\%) } \\
\text { - Mâles } \\
\text { - Génisses } \\
\text { Santé au 1er vêlage } \\
\text { Mortalité des veaux (\%) } \\
\text { Rétentions placentaires (\%) } \\
\text { Soins après le ler vêlage (\%) } \\
\text { Mammites (\%) } \\
\text { Production laitière (kg/j) } \\
\text { initiale (observée) } \\
\text { Ajustée (2) } \\
\text { maximale (observée) } \\
\text { Ajustée (2) } \\
\text { en } 16 \text { semaines (observée) } \\
\text { Ajustée (2) }\end{array}$ & $\begin{array}{r}15,4 \\
5,8 \\
21,2 \\
7,7 \\
12,5 \\
12,8 \\
19,2 \\
18,8 \\
17,0 \\
16,6\end{array}$ & & $\begin{array}{r}280,2 \\
37,3 \\
34,8 \\
\\
7,8 \\
7,3\end{array}$ & & $\begin{array}{l}14,5 \\
13,4 \\
20,8 \\
18,2 \\
18,5 \\
16,1\end{array}$ & & $\begin{array}{r}281,4 \\
\\
39,8 \\
36,5 \\
\\
8,3 \\
7,4 \\
\\
16,7 \\
4,2 \\
25,0 \\
8,3 \\
\\
14,8 \\
13,5 \\
22,2 \\
19,2 \\
20,0 \\
17,3\end{array}$ & \\
\hline Poids adulte (kg) (3) & 632 & a & 612 & $\mathrm{~b}$ & 611 & $\mathrm{~b}$ & 650 & $a b$ \\
\hline
\end{tabular}

(1) Les valeurs indexées d'une lettre différente sont statistiquement différentes $(\mathrm{P}<0,05)$.

(2) Productions laitières ajustées par analyse de variance : domaine, race, période de naissance, profil.

(3) Poids vif le plus élevé mesuré dans la semaine suivant le vêlage des vaches réalisant au moins 3 lactations.

vêlant à 2 ans ont gagné $487 \mathrm{~g} / \mathrm{j}$ contre $386 \mathrm{~g} / \mathrm{j}$ pour celles vêlant à 3 ans. Le poids vif après le premier vêlage des vaches primipares précoces a été de $479 \mathrm{~kg}$ contre $562 \mathrm{~kg}$ pour les vaches primipares tardives. Les productions laitières observées au cours des 16 premières de la lactation ont été peu différentes mais les productions ajustées après une analyse de variance montrent une production supplémentaire d'environ $2 \mathrm{~kg} / \mathrm{j}$ en faveur des vaches primipares tardives $(\mathrm{P}<0,01)$. Les veaux produits par les vaches primipares précoces ont été plus légers d'environ $3 \mathrm{~kg}(\mathrm{P}<0,01)$ mais plus lourds relativement au poids de la mère après le vêlage $(+0,6 \%$ environ, $\mathrm{P}<0,01)$. Ainsi, la mortalité des veaux issus de vaches primipares précoces s'est accrue de $50 \%(\mathrm{P}<0,05)$ et les cas de soins génitaux aux mères après le premier vêlage multipliés par 3,5 $(\mathrm{P}<0,01)$. La fréquence des mammites après le premier vêlage a été plus élevée chez les vaches primipares précoces $(+60 \%, \mathrm{P}<0,05)$.

\section{1 / Description des profils de croissance}

\section{a / Vêlages à 2 ans}

Pour ces génisses primipares précoces, 4 profils (48 à 117 génisses par profil) ont été retenus (tableau 2, figure 2). Les gains de poids vif ont varié de $627 \mathrm{~g} / \mathrm{j}$ (C2) à $806 \mathrm{~g} / \mathrm{j}$ (B2) de la naissance à l'âge de 6 mois, de $560 \mathrm{~g} / \mathrm{j}$ (D2) à $810 \mathrm{~g} / \mathrm{j}(\mathrm{A} 2)$ entre les âges de 6 et 12 mois et de $401 \mathrm{~g} / \mathrm{j}(\mathrm{A} 2)$ à $601 \mathrm{~g} / \mathrm{j}$ (D2) de l'âge de 12 mois à après le premier vêlage. Les génisses D2 les plus légères à l'âge de 12 mois étaient les plus jeunes (23,7 mois) et les plus lourdes après le premier vêlage $(489 \mathrm{~kg})$. L'écart maximal de poids vif après le premier vêlage a été de $19 \mathrm{~kg}$ (C2 et D2, NS). Aux âges de 6 et 12 mois, les plus grands écarts ont été respectivement de $31 \mathrm{~kg}$ (B2 et $\mathrm{C} 2$ ) et $50 \mathrm{~kg}$ (A2 et D2). 
Figure 2. Evolution du poids vif des génisses selon les profils.

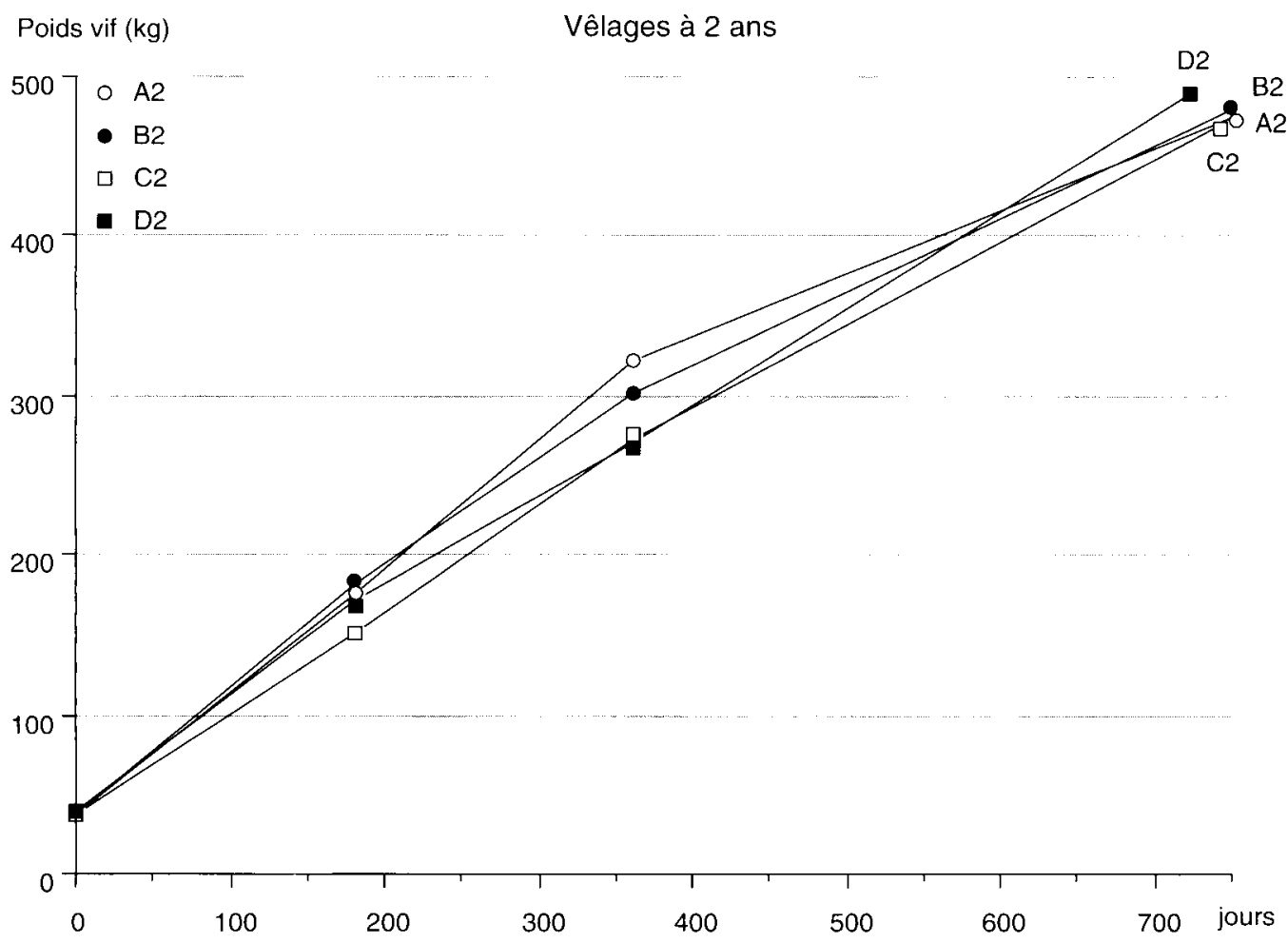

Vêlages à 3 ans

Poids vif $(\mathrm{kg})$

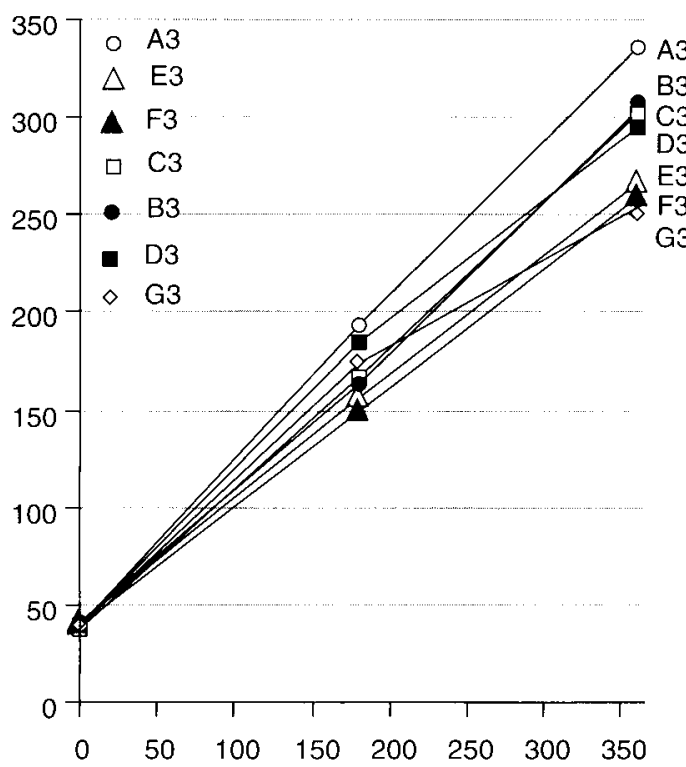

\section{b / Vêlages à 3 ans}

Pour ces génisses, 7 profils (48 à 131 génisses par profil) ont été retenus (tableau 3, figure 2). Les gains de poids vif ont varié de $616 \mathrm{~g} / \mathrm{j}$ (F3) à $869 \mathrm{~g} / \mathrm{j}$ (A3) de la naissance à l'âge de 6 mois, de $440 \mathrm{~g} / \mathrm{j}$ (G3) à $783 \mathrm{~g} / \mathrm{j}$ (B3) entre les âges de 6 et 12 mois et de $316 \mathrm{~g} / \mathrm{j}$ (C3) à $461 \mathrm{~g} / \mathrm{j}$ (E3) de l'âge de 12 à après le premier vêlage. Aux âges de 6 mois, 12 mois et après le premier vêlage, les écarts maximaux de poids vifs ont été respectivement de $46 \mathrm{~kg}$ (A3 et F3), de $81 \mathrm{~kg}$ (A3 et G3) de $94 \mathrm{~kg}$ (A3 et F3). Les génisses E3 et G3 légères à l'âge de 1 an ont eu des gains de

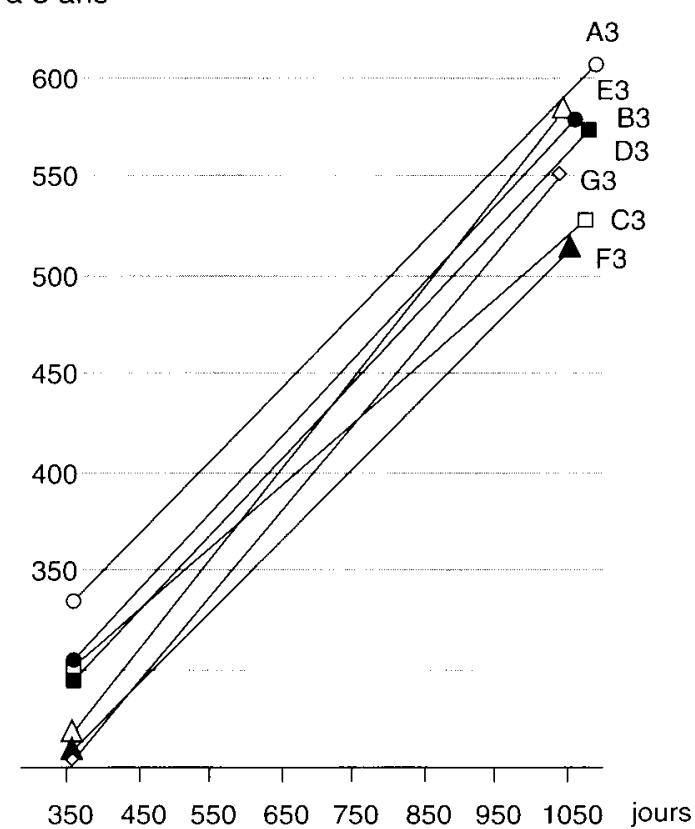

poids vif élevés de l'âge de 1 an à après le premier vêlage $(450 \mathrm{~g} / \mathrm{j})$. Par contre, les génisses C3 et F3 ont eu un gain de poids vif faible sur la même période compte tenu de leur poids vif faible ou modéré à l'âge de 1 an.

Les vaches $A 3$ et $E 3$ ont eu des gains de poids vifs déséquilibrés avant et après l'âge de 1 an $(\mathrm{A} 3: 824$ et $371 \mathrm{~g} / \mathrm{j}, \mathrm{E} 3: 630$ et $461 \mathrm{~g} / \mathrm{j})$. Les gains de poids vif des génisses B3 et D3 ont été moyens $(720 \mathrm{~g} / \mathrm{j}$ de la naissance à l'âge de 1 an et $390 \mathrm{~g} / \mathrm{j}$ ensuite). Cependant, les génisses B3 ont eu un gain de poids plus faible de la naissance à l'âge de 6 mois $(687 \mathrm{~g} / \mathrm{j})$ et 
Tableau 3. Performances des génisses et vaches primipares tardives selon les profils (1).

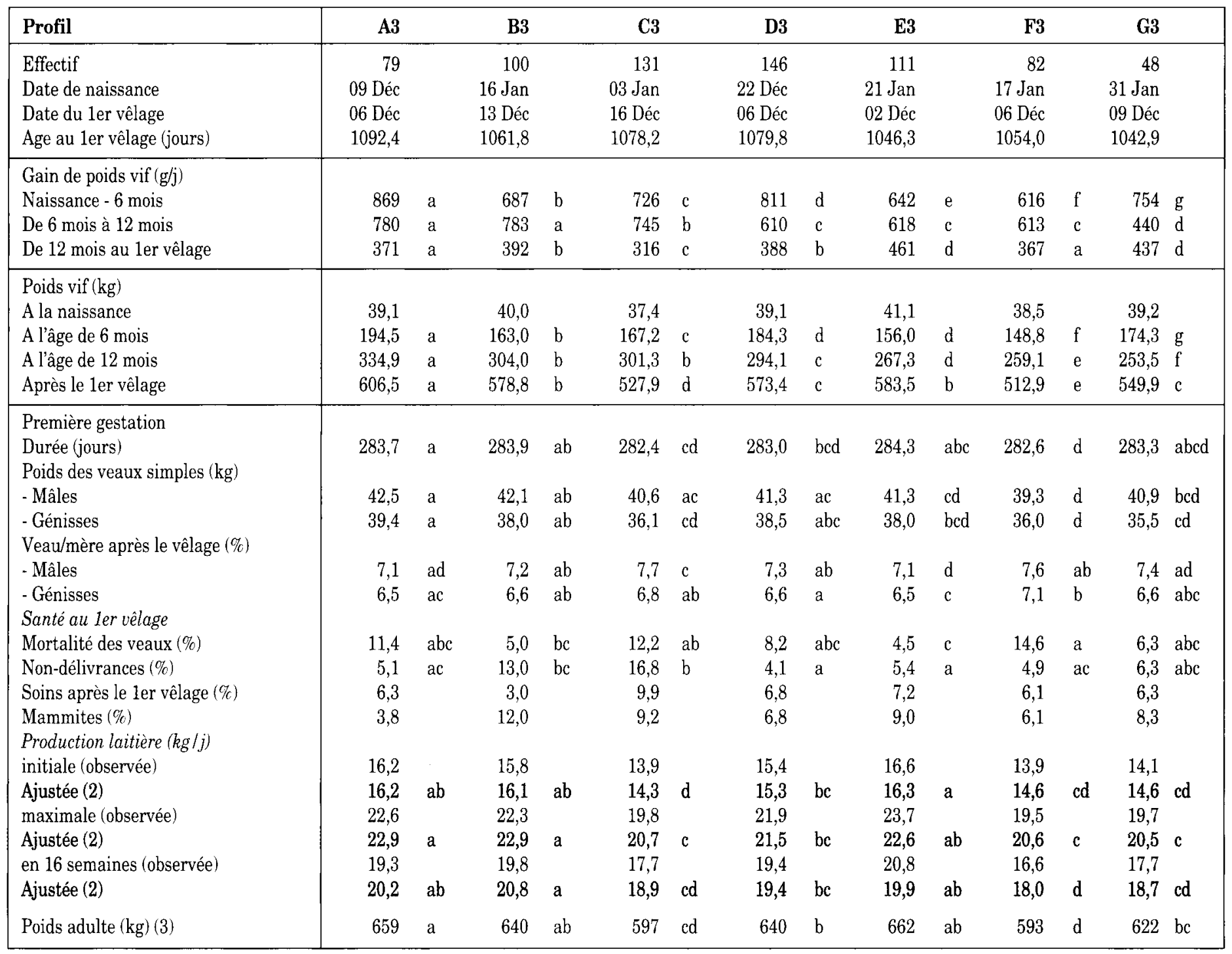

(1) Les valeurs indexées d'une lettre différente sont statistiquement différentes $(\mathrm{P}<0,05)$.

(2) Productions laitières ajustées par analyse de variance : domaine, race, saison de naissance, profil.

(3) Poids vif le plus élevé mesuré dans la semaine suivant le vêlage des vaches réalisant au moins 3 lactations.

plus élevé entre les âges de 6 et 12 mois $(783 \mathrm{~g} / \mathrm{j})$. C'est l'inverse pour les génisses D3 avec un déséquilibre net des croissances : $811 \mathrm{~g} / \mathrm{j}$ de la naissance à 6 mois et $610 \mathrm{~g} / \mathrm{j}$ entre les âges de 6 et 12 mois.

\section{2 / Analyse des profils de croissance}

La répartition des génisses dans les profils de croissance diffère selon leurs caractéristiques (tableau 4). L'analyse globale des relations entre ces profils et ces caractéristiques a permis de dégager 3 groupes constitutifs essentiels pour les primipares précoces et 4 groupes pour les primipares tardives (tableau 4). Pour les vaches précoces, 2 groupes ont des effectifs importants, l'un de génisses de Marcenat et l'autre de génisses de Theix. Les profils C2 et D2 sont constitués presque exclusivement de génisses pie noir élevées à Theix, alors que les profils $\mathrm{A} 2$ et $\mathrm{B} 2$ se répartissent de manière homogène entre les
2 domaines. Pour les primipares tardives, les génisses se répartissent en 2 groupes dans chacun des 2 domaines de Marcenat et Orcival. Les profils A3, B3 et C3 sont caractéristiques du domaine de Marcenat, les animaux du profil A3 étant nés plutôt en automne et ceux du profil B3 plutôt au printemps et après 1971 . Les profils D3 et E3 sont caractéristiques du domaine d'Orcival et se distinguent par la période de naissance (automne pour D3, hiver pour E3). Les profils F3 et G3, en tout 130 génisses, sont équitablement répartis.

\section{3 / Profils de croissance et performances au début de la première lactation}

Lorsque les conditions de naissance des génisses (domaine, race, période de naissance, saison de naissance) sont prises en compte, les écarts de production laitière en début de première lactation se réduisent sensiblement d'un 
Tableau 4. Répartition dans les profils des génisses selon leurs caractéristiques (A).

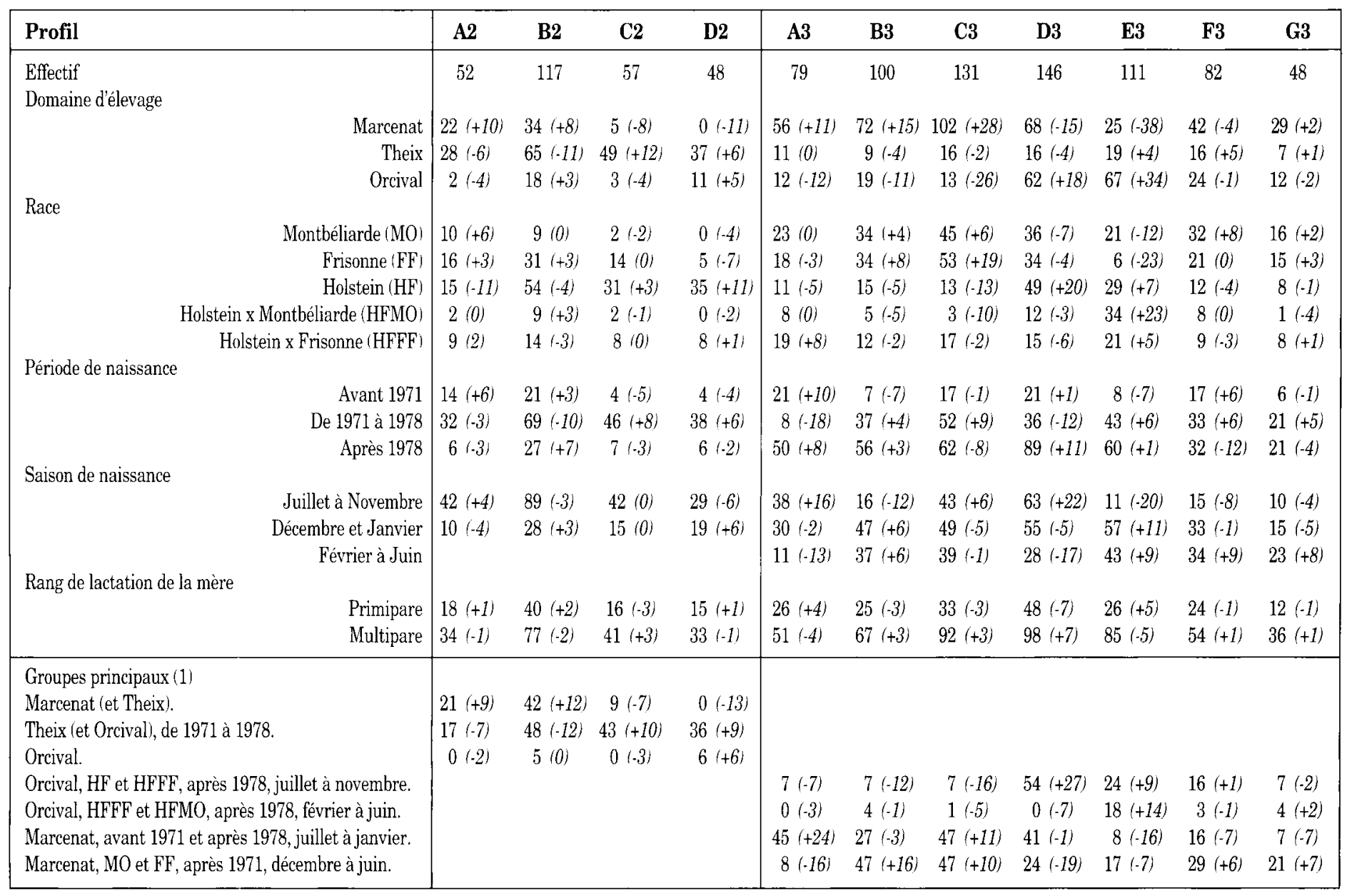

(1) Principaux groupes d'animaux définis par une procédure de classification entre les profils et les caractéristiques des animaux (au moins 10 animaux par cellule croisant le domaine, la race, la période et la saison de naissance).

(A) Entre parenthèses, les écarts aux effectifs théoriques pour une répartition équilibrée des génisses dans les profils selon les conditions de naissance (domaine, race, période et saison de naissance, rang de lactation de la mère, groupe).

profil à l'autre, aussi bien pour les vêlages précoces (tableau 2) que pour les vêlages tardifs (tableau 3). Les productions laitières ajustées sont identiques pour les vaches primipares précoces des 4 profils. Parmi les profils des vêlages tardifs, les vaches A3, B3 et E3 (579 à $606 \mathrm{~kg}$ de poids vif) ont eu une production ajustée de lait en 16 semaines supérieure de $1,8 \mathrm{~kg} / \mathrm{j}(\mathrm{P}<0,01)$ à celles des vaches $\mathrm{C} 3, \mathrm{~F} 3$ et G3 (528 à $550 \mathrm{~kg}$ ); les vaches D3 ont eu une production intermédiaire malgré un poids vif élevé après le premier vêlage ( $573 \mathrm{~kg})$. Les vaches B3 $(579 \mathrm{~kg})$ sont celles qui ont produit le plus $(20,8 \mathrm{~kg} / \mathrm{j})$ et les vaches F3 $(513 \mathrm{~kg})$ celles qui ont produit le moins de lait $(18,0 \mathrm{~kg})$.

Les fréquences des affections sanitaires n'ont pas été différentes selon les profills des vaches primipares précoces. La mortalité des veaux issus des vaches primipares tardives a été plus élevée parmi les vaches A3 (génisses les plus lourdes), C3 et F3 (veaux les plus lourds relativement à leur mère) par rapport aux autres $(P<0,1)$. Les rétentions placentaires sont survenues plus fréquemment $(\mathrm{P}<0,01)$ chez les vaches des profils B3 $(13,0 \%)$ et C3 $(16,8 \%)$ que chez celles des autres profils $(5 \%)$.

\section{3 / Discussion}

Les profils de croissance des génisses vêlant à deux ans observés dans cette étude sont peu différents. En fait, les niveaux de croissance sont très déterminés pour les vêlages de ce type. Des croissances trop faibles dans le jeune âge conduisent à repousser la mise à la reproduction, ou à accélérer la croissance ultérieure via l'alimentation. Les génisses D2 entrent en partie dans ce dernier schéma mais il s'agit aussi d'animaux d'un potentiel de croissance élevé, comme en témoigne leur format adulte important. Les poids atteints au premier vêlage étant en moyenne très voisins d'un profil à l'autre, il est logique que les performances de production en première lactation des vaches de ces différents profils soient très comparables. En effet, la production laitière en première lactation est largement déterminée par le poids au premier vêlage (Lee 1976, Amir et al 1978, Foldager et Sejrsen 1991).

Les vaches vêlant pour la première fois à trois ans représentent certainement une population moins "pilotée". Les différences de poids au premier vêlage atteignent près de $100 \mathrm{~kg}$ 
entre les profils extrêmes. Les écarts sont sensibles dès l'âge de six mois. Le domaine (et donc la race, qui lui est très liée) et la période de naissance sont les facteurs principaux qui permettent de discriminer de manière globale les profils. Cependant, ils ne permettent pas d'opposer de manière cohérente les profils extrêmes. Ainsi, les profils A3 et E3 conduisent à des poids élevés et voisins au premier vêlage mais sont associés à des domaines et à des périodes de naissance différents. A l'opposé, les génisses C3 et F3 présentent des croissances faibles pour toute la période d'élevage or les génisses C3 sont nées aussi bien à l'automne qu'au printemps, et les génisses F3 sont équitablement réparties entre les domaines et les périodes de naissance. Ces profils sont donc déterminés soit par des pratiques d'élevage, non connues dans cette étude, soit par les caractéristiques génétiques des animaux. En particulier, les différences d'âge à la puberté et les phénomènes de croissance compensatrice n'ont pas pu être pris en compte.

Contrairement au cas des vêlages précoces, les profils des vêlages tardifs ont des conséquences sensibles sur les performances en première lactation, même lorsqu'on tient compte des conditions de naissance. Ce sont les profils conduisant aux poids au premier vêlage les plus élevés (A3, B3, E3) qui sont associés aux productions laitières ajustées les plus fortes, et ce indépendamment des croissances intermédiaires observées. Ainsi, les profils A3 et B3, qui présentent des croissances différentes de la naissance à l'âge de 6 mois (respectivement 869 et $687 \mathrm{~g} / \mathrm{j}$ ) ont des productions voisines en première lactation. Cette absence d'effet important des croissances intermédiaires sur la production laitière est vraisemblablement due au fait qu'elles se situent dans la gamme recommandée compte tenu du format adulte des vaches (Troccon 1993). Cependant, l'optimum de croissance au regard de la production laitière ultérieure est plus souvent situé entre 600 et $800 \mathrm{~g} / \mathrm{j}$ au cours de la période prépubère (Amir et Kali 1975, Foldager et Sejrsen 1991).

Les profils des vêlages tardifs sont aussi associés à des différences d'état sanitaire au début de la première lactation. Les profils qui se ressemblent par leur association avec l'état sanitaire peuvent pourtant être bien différents à terme (par exemple B3 et C3 à l'égard des rétentions placentaires). Ici, les problèmes sanitaires sont plutôt associés aux profils de génisses à faible développement corporel au premier vêlage.

\section{Conclusion}

Les gains de poids vif à rechercher pour des génisses primipares tardives sont de $700 \mathrm{~g} / \mathrm{j}$ de la naissance à l'âge de 1 an et de $400 \mathrm{~g} / \mathrm{j}$ de 1 an à après le premier vêlage $(500 \mathrm{~g} / \mathrm{j}$ avec l'ensemble foeto-placentaire). Les poids vifs de ces génisses (40 kg à la naissance) seraient de $170 \mathrm{~kg}$ à l'âge de 6 mois, $300 \mathrm{~kg}$ à l'âge de 1 an et $600 \mathrm{~kg}$ après le premier vêlage. Ces poids vifs représentent des objectifs d'environ 25,45 et $90 \% \mathrm{du}$ poids adulte $(675 \mathrm{~kg})$ aux âges de 6 mois, 12 mois et après le premier vêlage ( 30 , 50 et $80 \%$ pour les génisses primipares précoces ; Troccon 1993). Si la conduite alimentaire des génisses après l'âge de 1 an ne permet pas d'atteindre le gain de poids vif de $500 \mathrm{~g} / \mathrm{j}$, l'objectif de croissance au cours de la première année sera révisé à la hausse $(800 \mathrm{~g} / \mathrm{j})$. Le poids vif à la mise à la reproduction $(27$ mois $)$ sera de $450-500 \mathrm{~kg}$.

Ces objectif's de croissance faibles après l'âge de 1 an offrent la possibilité de réduire l'apport d'aliment concentré (céréales), de valoriser des fourrages pauvres et/ou la pousse de l'herbe pendant l'hiver, d'obtenir une croissance compensatrice des génisses au pâturage le printemps suivant et de limiter leur engraissement, défavorable à la longévité des vaches (Troccon et Petit 1989). La croissance compensatrice est un phénomène très documenté mais très variable selon l'intensité de la restriction et sa durée, et aussi selon la qualité du fourrage offert pendant la phase de réalimentation (Ryan 1990). Selon O'Donovan (1984), elle varie de la moitié aux deux-tiers mais nous observons des taux plus élevés sur des prairies de ray-grass anglais en Bretagne et en Normandie.

\section{Remerciements}

Nous tenons à remercier l'ensemble des personnels de Theix, Orcival et Marcenat qui ont réalisé la collecte de ces données, et en particulier A. Ollier, J. Rouel, J. Bony et J.P. Garel pour leur aide dans leur dépouillement. Merci à B. Rémond pour ses suggestions fort utiles sur le manuscrit. 


\section{Références bibliographiques}

Amir S., Kali J., 1975. Some topics for future research. In "The early calving of heifers and its impact on beef production", Tayler J.C. (Editor), 274-280.

Amir S., Kali J., Volcani R., 1968. Influence of growth rate on reproduction and lactation in dairy cattle. In "Growth and Development of Mammals", Lodge G.A. and Lamming G.E. (Editors), 234-263.

Amir S., Halevi A., Edelman Z., Kali J., 1978. The effect of age at first calving on subsequent milk yield of dairy cows. Volcani Center, Bet Dagan, Israël, special publication $n^{\circ} 119$.

Capuco A.V., Smith J.J., Waldo D.R., Elsasser T.H., 1988. Effect of diet and prepubertal growth rate of Holstein heifers on mammary gland and milk production. J. Dairy Sci., 71 ( suppl. 1), 229 (Abst.).

Coulon J.B., Lescourret Françoise, Faye B., Landais E., Troccon J.L., Perochon L., 1993. Description de la base de données "LASCAR", un outil pour l'étude des carrières des vaches laitières. INRA Prod. Anim., 6 (2), 151-160.

Ducker M.J.R., Haggett R.A., Fisher W.J., Morant S.V., Bloomfoeld G.A., 1985. Nutrition and reproductive performance of dairy cattle. I. The effect of level of feeding in late pregnancy and around the time of insemination on the reproductive performance of first lactation dairy heifers. Anim. Prod., 41, 1.

Foldager J., Sejrsen K., 1991. Rearing intensity in dairy heifers and the effect on subsequent milk production. 693. Beretning fra Statens Husdyrbrugsforsog, Copenhagen, $131 \mathrm{pp}$.

Hansson A., Brannang E., Liljedahl L.E., 1967 Studies on monozygous cattle twins. XIX. The interaction of heredity and intensity of rearing with regard to growth and milk yield in dairy cattle. Lantbr. H’gsk. Ann., 33, 643-649.

Harrisson R.D., Reynolds L.P., Little W., 1973. A quantitative analysis of mammary glands of dairy heifers reared at different rates of live-weight gain. J. Dairy Res., 50, 405-412.
Jammes Hélène, Djiane J., 1988. Le développement de la glande mammaire et son contrôle hormonal dans l'espèce bovine. INRA Prod. Anim., 1 (5), 299310.

Lacasse P., Block E., Guilbaut L.A., Petitclerc D., 1993. Effect of plane of nutrition of dairy heifers before and during gestation on milk production, reproduction and health. J. Dairy Sci., 76 (11), 3420-3247.

Lee A.J., 1976. Relationship between milk yield and age at calving in first lactation. J. Dairy Sci., 59, 1794-1801.

Little W., Kay R.M., 1979. The effects of rapid growth and early calving on the subsequent performance of dairy heifers. Anim. Prod., 29, 131-142.

O'Donovan P.PB., 1984. Compensatory gain in cattle and sheep. Nutrition Abstracts and Reviews. Series B, Livestock Feeds and Feeding, 54, 389-410.

Ryan W.J., 1990. Compensatory growth in cattle and sheep. Nutrition Abstracts and Reviews. Series B, Livestock Feeds and Feeding, 60, 653-664.

Sejrsen K., Huber J.T., Tucker H.A., 1983. Influence of amount fed on hormone concentrations and their relationship to mammary growth in heifers. J. Dairy Sci., 66 (4), 845-855.

Sinha Y.N., Tucker H.A., 1969. Mammary development and pituitary prolactin levels of heifers from birth through puberty and during the oestrus. J. Dairy Sci., 52, 507-512.

Statistical Application Systems, 1987. SAS/STAT Guide for Personnal Computers, Version 6 Edition. Cary, NC, SAS Institute Inc., 1028 pp.

Troccon J.L., 1993. Effets de l'alimentation hivernale des génisses sur les performances et la longévité des vaches. INRA Prod. Anim., 6 (5), 345-356.

Troccon J.L., Petit M., 1989. Croissance des génisses de renouvellement et performances ultérieures. INRA Prod. Anim., 2, 55-64.

Waldo D.R., Rexroad C.E., Capuco A.V., 1988 Effects of diet and daily gain on milk production of Holstein cattle. J. Dairy Sci., 71 (suppl 1), 217 (Abst.). 


\section{Summary}

Productive life of the dairy cow : effect of breeding period on performances in first lactation.

Growth curves from 971 heifers born from 1963 to 1990 in 3 experimental INRA herds were analysed as well as their consequences on performances in early first lactation. These heifers from Montbéliarde, Friesian and Holstein breeds and their Holstein crossbreeds had early (2 years old, $\mathrm{n}=274$ ) or late (3 years old, $\mathrm{n}=697$ ) calvings. Early primiparous cows produced less milk $(2 \mathrm{~kg} / \mathrm{d})$, and had more health disorders at calving than late ones. These 2 groups were analysed separately.

A classification of these heifers based on their liveweight gains (birth to 6 months, 6 to 12 months, 12 months to first calving) allowed different profiles possible to be built. These profiles were analysed according to herd, breed, birth period and birth season. Post-calving live-weights varied from 470 to $490 \mathrm{~kg}$ for early calving heifers and from 510 to $610 \mathrm{~kg}$ for late calving heifers according to the profiles. The largest live-weight differences were $45 \mathrm{~kg}$ and $81 \mathrm{~kg}$ at 6 and 12 months. Early calving profiles $(n=4)$ had no effect on milk production in early first lactation. Late calving heifers (7 profiles) produced more milk when post-calving weight increased $(+1.8 \mathrm{~kg} / \mathrm{d}$ from $530 \mathrm{~kg}$ to $590 \mathrm{~kg}$ ). Moderate live-weight gains are suitable for late calving heifers from birth to 12 months $(700 \mathrm{~g} / \mathrm{d}$ ) and then to post-calving age $(400 \mathrm{~g} / \mathrm{d})$. These values agree with results observed in heifers in this study.

TROCCON J.L., COULON J.B., LESCOURRET F., 1994. Carrière des vaches laitières : caractérisation de la phase d'élevage et relation avec les performances en première lactation. INRA Prod. Anim. 7 (5), 359-368.

\section{Annexe 1}

\section{Génisses jumelles ( $n=56$ )}

Les 56 génisses jumelles sont essentiellement des filles de vaches multipares $(n=44)$ de race Montbéliarde $(n=22)$ ou de croisement Holstein x Montbéliard $(n=11)$ nées à Marcenat $(n=28)$ ou à Orcival $(n=19)$ après $1978(n=37)$. Leur poids à la naissance est inférieur $(32,6 \mathrm{~kg})$ à celui des génisses nées simples $(38,7 \mathrm{~kg})$. Leurs croîts en élevage et leurs performances en première lactation sont comparables à celles des génisses nées simples.

\section{Génisses à gestation gémellaire (n=39)}

Les 15 génisses portant des veaux jumeaux pour un vêlage vers 2 ans ont une gestation écourtée de 20 jours. Le gain de poids vif entre l'âge de 1 an et après la première parturition est réduit de $90 \mathrm{~g} / \mathrm{j}$ par rapport aux génisses ne portant qu'un veau. Leur poids vif après le premier vêlage et leur production laitière en première lactation sont plus faibles de $55 \mathrm{~kg}$ et $\mathrm{de} 4,2 \mathrm{~kg} / \mathrm{j}$ comparativement aux génisses à gestation simple. Ces différences sont atténuées pour les 24 génisses vêlant vers l'âge de 3 ans et produisant des jumeaux. Le manque de développement, l'insuffisance d'état et le manque de préparation à la lactation sont sans doute à l'origine de cette faible production laitière.

\section{Effectif}

Age au ler vêlage (mois)

Gain de poids vif $(g / j)$

Naissance -6 mois

6 mois - 12 mois

12 mois - après 1er vêlage

Poids vif ( $k g$ )

Naissance

6 mois

12 mois

Après ler vêlage

Durée de gestation (j)

Production laitière $(\mathrm{kg} / \mathrm{j})$

- initiale

- maximale

- 16 semaines

$\begin{array}{cc}\text { Génisses } & \text { nées jumelles } \\ 10 & 46 \\ 24,2 & 35,1 \\ & \\ 748 & 779 \\ 661 & 601 \\ 528 & 399 \\ & \\ 32,1 & 32,7 \\ 166,0 & 172,2 \\ 285,0 & 280,3 \\ 481,7 & 560,8 \\ 279,8 & 284,3 \\ & \\ 14,5 & 14,9 \\ 22,3 & 22,0 \\ 19,3 & 19,7\end{array}$

\begin{tabular}{|c|c|}
\hline \multicolumn{2}{|c|}{ Gestations gémellaires } \\
\hline 15 & 24 \\
\hline 23,3 & 34,7 \\
\hline 714 & 753 \\
\hline 683 & 714 \\
\hline 398 & 341 \\
\hline 37,7 & 38,6 \\
\hline 165,5 & 173,4 \\
\hline 288,3 & 301,9 \\
\hline 424,1 & 538,8 \\
\hline 256,4 & 276,0 \\
\hline 9,7 & 13,2 \\
\hline 16,9 & 20,3 \\
\hline 14,6 & 18,2 \\
\hline
\end{tabular}

